\title{
Identities for generalized fractional integral operators associated with products of analogues to Dirichlet averages and special functions
}

\author{
Hemant Kumar $^{1 *}$, M. A. Pathan ${ }^{2}$ and Shilesh Kumari ${ }^{3}$ \\ ${ }^{1 * \& 3}$ Department of Mathematics, D. A-V. P. G. College, Kanpur, (U. P.), INDIA \\ ${ }^{2}$ Department of Mathematics, University of Botswana, Private Bag 0022, Goborone, BOTSWANA, \\ *Corresponding Authors - e-mails: palhemant2007@rediffmail.com (Hemant Kumar and Shilesh Kumari); mapathan@gmail.com (M. A. Pathan)
}

\begin{abstract}
In this present work an attempt has been made to define two generalized fractional integral operators associated with products of analogues to Dirichlet averages and special functions. Discussions on the different aspects of the obtained results have been followed by utilization in finding out the images of multivariate function involving multivariate G-function. We make their applications in statistics also.
\end{abstract}

Keywords: Analogues to Dirichlet averages, generalized fractional integral operators, identities, multivariable G-function, special functions.

(2000) MSC : 28A25, 33C60, 33C65, 44A20, 44A30, 44A99, 47G10.

\section{Introduction}

The generalized fractional integral operators have introduced in the form (Saigo and Maeda, 1996):

$$
\left(I_{0^{+}}^{a, a^{\prime}, b, b^{\prime}, c} f\right)(x)=\frac{x^{-a}}{\Gamma(c)} \int_{0}^{x}(x-t)^{c-1} t^{-a^{\prime}} F_{3}\left[a, a^{\prime}, b, b^{\prime} ; c ; 1-\frac{t}{x}, 1-\frac{x}{t}\right] f(t) d t
$$

and

$$
\left(I_{0^{-}}^{a, a^{\prime}, b, b^{\prime}, c} f\right)(x)=\frac{x^{-a^{\prime}}}{\Gamma(c)} \int_{x}^{\infty}(t-x)^{c-1} t^{-a} F_{3}\left[a, a^{\prime}, b, b^{\prime} ; c ; 1-\frac{x}{t}, 1-\frac{t}{x}\right] f(t) d t
$$

provided that a, a', b, b', c are complex, $\mathrm{x}>0, \operatorname{Re}(\mathrm{c})>0, \mathrm{~F}_{3}[\cdot]$ is one of the Appell functions of two variables (Appell and Kampé de Fériet, 1926 and Erdélyi et al., 1953, p.224 eq. (8)), $f(\mathrm{t})$ is integrable in the interval $(0, \infty)$ and the Gamma function is defined by $\Gamma(\lambda+n)=(\lambda)_{n} \Gamma(\lambda), n \geq 0, \lambda \neq 0$.

Particularly, for $a=\alpha+\beta, c=\alpha, b=-v$ and $a^{\prime}=0$ such that $\operatorname{Re}(\alpha)>0$, the equations (1.1) and (1.2) yield Saigo operators in the form (Saigo, 1978)

$$
\left(I_{0^{+}}^{\alpha+\beta, 0,-v, b^{\prime}, \alpha} f\right)(x)=\left(I^{\alpha, \beta, v} f\right)(x)=\frac{x^{-\alpha-\beta}}{\Gamma(\alpha)} \int_{0}^{x}(x-t)^{\alpha-1}{ }_{2} F_{1}\left[-v, \alpha+\beta ; \alpha ; 1-\frac{t}{x}\right] f(t) d t
$$

and

$$
\left(I_{0^{-}}^{\alpha+\beta, 0,-v, b^{\prime}, \alpha} f\right)(x)=\left(J^{\alpha, \beta, v} f\right)(x)=\frac{1}{\Gamma(\alpha)} \int_{x}^{\infty}(t-x)^{\alpha-1} t_{2}^{-\alpha-\beta}{ }_{2} F_{1}\left[-v, \alpha+\beta ; \alpha ; 1-\frac{x}{t}\right] f(t) d t
$$

respectively, ${ }_{2} \mathrm{~F}_{1}[$.$] is the Gaussian hypergeometric function (Rainville, 1971, and Erde'lyi et al., 1953, p.58 eq. (2) ))$ 
Again, for $\alpha+\beta=0$, or,$\beta=0$, and $\operatorname{Re}(\alpha)>0$, the equations (1.3) and (1.4) are converted into following operators (Kilbas et al., 2006, Samko et al., 1993, Kiryakova, 2006)

$\left(I^{\alpha,-\alpha, v} f\right)(x)=\frac{1}{\Gamma(\alpha)} \int_{0}^{x}(x-t)^{\alpha-1} f(t) d t=R_{t}^{\alpha}\{f(t)\}(x) \quad$ (Riemann-Liouville operator)

or

$\left(I^{\alpha, 0, v} f\right)(x)=\frac{x^{-\alpha-v}}{\Gamma(\alpha)} \int_{0}^{x}(x-t)^{\alpha-1} t^{v} f(t) d t=E_{t}^{\alpha, v}\{f(t)\}(x) \quad$ (Erde'lyi-Kober operator)

and

$\left(J^{\alpha,-\alpha, v} f\right)(x)=\frac{1}{\Gamma(\alpha)} \int_{x}^{\infty}(t-x)^{\alpha-1} f(t) d t=W_{t}^{\alpha}\{f(t)\}(x) \quad$ (Weyl operator)

or

$\left(J^{\alpha, 0, v} f\right)(x)=\frac{x^{v}}{\Gamma(\alpha)} \int_{x}^{\infty}(t-x)^{\alpha-1} t^{-\alpha-v} f(t) d t=K_{t}^{\alpha, v}\{f(t)\}(x) \quad$ (Erde'lyi-Kober operator)

respectively.

The Dirichlet average is defined by (Carlson, 1977, Gupta and Agrawal, 1990)

$F(b ; z)=\int_{E} g(u . z) d \mu_{b}(u), u . z=\sum_{i=1}^{k} u_{i} z_{i}, 0 \leq u_{i} \leq 1, \ldots, 0 \leq u_{k-1} \leq 1, u_{k}=1-u_{1}-\ldots-u_{k-1}$

and $\mathrm{g}$ is measurable on the standard simplex $\mathrm{E}$ in $R^{k}, k \geq 2$ and $\mathrm{F}(\mathrm{b} ; \mathrm{z})=\mathrm{g}(\mathrm{z})$, when $\mathrm{k}=1$

and

$d \mu_{b}(u)=\frac{1}{B(b)} u_{1}^{b_{1}-1} \ldots u_{k-1}^{b_{k-1}-1}\left(1-u_{1}-\ldots-u_{k-1}\right)^{b_{k}-1} d u_{1} \ldots d u_{k-1}$

The function $\mathrm{B}(\mathrm{b})$ is given by

$B(b)=\frac{\Gamma\left(b_{1}\right) \ldots \Gamma\left(b_{k}\right)}{\Gamma\left(b_{1}+\ldots+b_{k}\right)}, b=\left(b_{1}, \ldots, b_{k}\right) \in C^{k}, \operatorname{Re}\left(b_{i}\right)>0, \forall i=1,2, \ldots, k$

The standard (k-1)-simplex (or unit (k-1)-simplex) $\mathrm{E}$ is the subset of $\mathrm{R}^{\mathrm{k}}$ and is given by

$E=\left\{\left(u_{1}, \ldots, u_{k}\right) \in R^{k}: \sum_{i=1}^{k} u_{i}=1\right.$ and $\left.u_{i} \geq 0, \forall i \in\{1,2, \ldots, k\}\right\}$

Now, in our investigation we introduce an integral average for a function g measurable on $\mathrm{E}$ in $R^{k}, k \geq 2$, identical to Dirichlet average defined in equations ((1.9)-(1.11)) (Carlson, 1997) defined by

$F\{g\}(b: z, x)=\int_{E} g\left(u_{1}+\ldots+u_{k}\right) d \mu_{b, z, x}(u)$

and $F\{g\}(b ; z, x)=\frac{z^{b}}{x^{b}} g(z)$, fork $=1$

where, $d \mu_{b, z, x}(u)$ is given by

$d \mu_{b, z, x}(u)=\frac{z_{k}^{b_{k}}}{B(b) x_{k}^{b_{k}}} u_{1}^{b_{1}-1} \ldots u_{k-1}^{b_{k-1}-1}\left(1-\frac{u_{1} x_{1}}{z_{1}}-\ldots-\frac{u_{k-1} x_{k-1}}{z_{k-1}}\right)^{b_{k}-1} d u_{1} \ldots d u_{k-1}$

in the region

$0 \leq u_{1} \leq \frac{z_{1}}{x_{1}} ; x_{1}>z_{1}>0, \ldots, 0 \leq u_{k-1} \leq \frac{z_{k-1}}{x_{k-1}} ; x_{k-1}>z_{k-1}>0, \frac{u_{k} x_{k}}{z_{k}}=1-\frac{u_{1} x_{1}}{z_{1}}-\ldots-\frac{u_{k-1} x_{k-1}}{z_{k-1}} ;$ 
$x_{k}>z_{k}>0, z=\left(z_{1}, \ldots, z_{k}\right)$ and $x=\left(x_{1}, \ldots, x_{k}\right) \in R^{k}$.

The second integral average for a function $\mathrm{g}$ measurable on $\mathrm{E}$ in $R^{k}, k \geq 2$, identical to Dirichlet average (Carlson, 1997) is defined by

$F *\{g\}(b: z, x)=\int_{E} g\left(u_{1}^{-1}+\ldots+u_{k}^{-1}\right) d \mu *_{b, z, x}(u)$

and $F *\{g\}(b: z, x)=\frac{x^{b}}{z^{b}} g(z)$, fork $=1$

where, $\mathrm{d} \mu{ }_{\mathrm{b}, \mathrm{z}, \mathrm{x}}(\mathrm{u})$ is given by

$d \mu *_{b, z, x}(u)=\frac{z_{k}^{-b_{k}} x_{k}^{b_{k}}}{B(b)} u_{1}^{-b_{1}-1} \ldots u_{k-1}^{-b_{k-1}-1}\left(1-\frac{z_{1}}{u_{1} x_{1}}-\ldots-\frac{z_{k-1}}{u_{k-1} x_{k-1}}\right)^{b_{k}-1} d u_{1} \ldots d u_{k-1}$

in the region

$$
\begin{aligned}
& \frac{z_{1}}{x_{1}} \leq u_{1}<\infty ; x_{1}>z_{1}>0, \ldots, \frac{z_{k-1}}{x_{k-1}} \leq u_{k-1}<\infty ; x_{k-1}>z_{k-1}>0, \frac{z_{k}}{u_{k} x_{k}}=1-\frac{z_{1}}{u_{1} x_{1}}-\ldots-\frac{z_{k-1}}{u_{k-1} x_{k-1}} ; \\
& x_{k}>z_{k}>0, z=\left(z_{1}, \ldots, z_{k}\right) \text { and } x=\left(x_{1}, \ldots, x_{k}\right) \in R^{k} .
\end{aligned}
$$

Motivated by above work, in this paper for any general special function

$\Phi\left(\alpha_{1}, \ldots, \alpha_{l}\right)=\sum_{r_{1}, \ldots, r_{l}=0}^{\infty} A_{r_{1}, \ldots, r_{l}} \alpha_{1}^{r_{1}} \ldots \alpha_{l}^{r_{l}}$

$A_{r_{1}, \ldots, r_{l}}$ is multiparametric coefficient real or complex and for $\alpha=\left(\alpha_{1}, \ldots, \alpha_{l}\right) \in C^{l}, l \geq k$ and $c=\left(c_{1}, \ldots, c_{k}\right) \in C^{k},\left(\alpha_{i}\right) \neq 0, \forall i=1,2, \ldots, l$ and $\operatorname{Re}\left(\mathrm{c}_{\mathrm{i}}\right)>0, \mathrm{i}=1,2, \ldots \mathrm{k}$, and the multivariable function $\Psi(., \ldots,$.$) is$ integrable in $\{(0, \infty)\}^{k}$, we define following generalized fractional integral operators involving products of analogous to Dirichlet average defined in the equations (1.12)-(1.14) and any general special function given in equation (1.18):

$$
\begin{aligned}
& H^{\alpha, b, c}\left\{g, \Psi\left(z_{1}, \ldots, z_{k}\right)\right\}\left(x_{1}, \ldots, x_{k}\right)=\frac{x_{1} \ldots x_{k}}{\Gamma\left(c_{1}\right) \ldots \Gamma\left(c_{k}\right)} \\
& \times \int_{0}^{x_{1}} \ldots \int_{0}^{x_{k}}\left(x_{1}-z_{1}\right)^{c_{1}-1} \ldots\left(x_{k}-z_{k}\right)^{c_{k}-1} z_{1}^{-c_{1}-1} \ldots z_{k}^{-c_{k}-1} F\{g\}(b ; z, x) \\
& \times \Phi\left(\alpha_{1} z_{1}^{-1}\left(x_{1}-z_{1}\right), \ldots, \alpha_{k} z_{k}^{-1}\left(x_{k}-z_{k}\right), \alpha_{k+1}, \ldots, \alpha_{l}\right) \Psi\left(z_{1}, \ldots, z_{k}\right) d z_{1} \ldots d z_{k}
\end{aligned}
$$

provided that all conditions given in the equations (1.12)-(1.14) are followed.

The second generalized fractional integral operators involving products of analogous to Dirichlet average defined in the equations (1.15)-(1.17) and any general special function given in equation (1.18):

$$
\begin{aligned}
& P^{\alpha, b, c}\left\{g, \Psi\left(z_{1}, \ldots, z_{k}\right)\right\}\left(x_{1}, \ldots, x_{k}\right)=\frac{x_{1}^{-c_{1}} \ldots x_{k}^{-c_{k}}}{\Gamma\left(c_{1}\right) \ldots \Gamma\left(c_{k}\right)} \\
& \times \int_{x_{1}}^{\infty} \ldots \int_{x_{k}}^{\infty}\left(z_{1}-x_{1}\right)^{c_{1}-1} \ldots\left(z_{k}-x_{k}\right)^{c_{k}-1} F *\{g\}(b ; z, x) \\
& \times \Phi\left(\alpha_{1} x_{1}^{-1}\left(z_{1}-x_{1}\right), \ldots, \alpha_{k} x_{k}^{-1}\left(z_{k}-x_{k}\right), \alpha_{k+1}, \ldots, \alpha_{l}\right) \Psi\left(z_{1}, \ldots, z_{k}\right) d z_{1} \ldots d z_{k}
\end{aligned}
$$

provided that all conditions given in the equations (1.15)-(1.17) are followed.

Particularly, setting $\mathrm{k}=1$ and $\mathrm{l}=2, A_{r_{1}, r_{2}}=\frac{(a)_{r_{1}}\left(a^{\prime}\right)_{r_{2}}(d)_{r_{1}}\left(d^{\prime}\right)_{r_{2}}}{(c)_{r_{1}+r_{2}} r_{1} ! r_{2} !}, \alpha=\left(\alpha_{1}, \alpha_{2}\right)$ 
such that $\alpha_{1}=-1$ and $\alpha_{2}=1-\frac{z}{x}$ and $\mathrm{g}(\mathrm{z})=\mathrm{z}^{1+\mathrm{c}-\mathrm{b}-\mathrm{a}} \mathrm{x}^{\mathrm{b}-\mathrm{a}^{-}-1}$ in equation (1.19) and then with the aid of equation (1.13), it becomes Saigo and Maeda operator defined in equation (1.1) such that

$H^{\alpha, b, c}\left\{z^{1+c-b-a} x^{b-a^{\prime}-1}, \Psi(z)\right\}(x)=\left(I_{0^{+}}^{a, a^{\prime}, d, d^{\prime}, c} \Psi\right)(x)$

Or setting k=1 and $1=2, A_{r_{1}, r_{2}}=\frac{(a)_{r_{1}}\left(a^{\prime}\right)_{r_{2}}(d)_{r_{1}}\left(d^{\prime}\right)_{r_{2}}}{(c)_{r_{1}+r_{2}} r_{1} ! r_{2} !}, \alpha=\left(\alpha_{1}, \alpha_{2}\right)$

such that $\alpha_{1}=-1$ and $\alpha_{2}=1-\frac{z}{x}$ and $\mathrm{b}=\mathrm{a}^{\prime}+1, \mathrm{~g}(\mathrm{z})=\mathrm{z}^{\mathrm{c}-\mathrm{a}^{\prime}-\mathrm{a}}$ in equation (1.19) and then with the aid of the equation (1.13), it becomes Saigo and Maeda operator defined in equation (1.1) such that

$H^{\alpha, a^{\prime}+1, c}\left\{z^{c-a^{\prime}-a}, \Psi(z)\right\}(x)=\left(I_{0^{+}}^{a, a^{\prime}, d, d^{\prime}, c} \Psi\right)(x)$

Again, setting $\mathrm{k}=1$ and $1=2, A_{r_{1}, r_{2}}=\frac{(a)_{r_{1}}\left(a^{\prime}\right)_{r_{2}}(d)_{r_{1}}\left(d^{\prime}\right)_{r_{2}}}{(c)_{r_{1}+r_{2}} r_{1} ! r_{2} !}, \alpha=\left(\alpha_{1}, \alpha_{2}\right)$

such that $\alpha_{1}=-1$ and $\alpha_{2}=1-\frac{x}{z}$ and $\mathrm{g}(\mathrm{z})=\mathrm{z}^{\mathrm{b}-\mathrm{a}^{\mathrm{t}}} \mathrm{x}^{-\mathrm{b}-\mathrm{a}+\mathrm{x}}$ in equation (1.20) and then with the aid of equation (1.15), it becomes Saigo and Maeda operator defined in equation (1.2) such that

$P^{\alpha, b, c}\left\{z^{b-a^{\prime}} x^{c-b-a}, \Psi(z)\right\}(x)=\left(I_{0^{-}}^{a, a^{\prime}, d, d^{\prime}, c} \Psi\right)(x)$

Or setting k=1 and $\mathrm{l}=2, A_{r_{1}, r_{2}}=\frac{(a)_{r_{1}}\left(a^{\prime}\right)_{r_{2}}(d)_{r_{1}}\left(d^{\prime}\right)_{r_{2}}}{(c)_{r_{1}+r_{2}} r_{1} ! r_{2} !}, \alpha=\left(\alpha_{1}, \alpha_{2}\right)$

such that $\alpha_{1}=-1$ and $\alpha_{2}=1-\frac{x}{z}, \mathrm{~b}=-\mathrm{a}+\mathrm{c}$ and $\mathrm{g}(\mathrm{z})=\mathrm{z}^{-\mathrm{a}+\mathrm{c}-\mathrm{a}^{\prime}}$ in equation (1.20) and then with the aid of the equation (1.15), it becomes Saigo and Maeda operator defined in equation (1.2) such that

$P^{\alpha, c-a, c}\left\{z^{c-a-a^{\prime}}, \Psi(z)\right\}(x)=\left(I_{0^{-}}^{a, a^{\prime}, d, d^{\prime}, c} \Psi\right)(x)$

Here in our work, we derive the identities for above generalized fractional integral operators defined in the equations (1.19) and (1.20) when $g\{z\}=(1-z)^{-\lambda}$,

$\lambda \in C, \operatorname{Re}(\lambda)>0$. Again, we make their applications to find out the images of a multivariable function consisting generalized multivariable G-function. Finally, we discuss some of their deductions and applications in statistics.

The generalized multivariable G-function is the particular case of following multivariable H-function defined in the multiple contour integrals (Srivastava and Panda, 1976a, 1976b; Srivastava, Gupta and Goyal, 1982):

$H_{A, C:\left[B^{(1)}, D^{(1)}\right] ; \ldots ;\left[B^{(k)}, D^{(k)}\right]}^{0, \lambda:\left(\mu^{(1)}, \nu^{(1)}\right) ; \ldots ;\left(\mu^{(k)}, v^{(k)}\right)}[$
$\left[(a): \theta^{(1)}, \ldots, \theta^{(k)}\right]:\left(\left(b^{(1)}\right):\left(\phi^{(1)}\right)\right) ; \ldots ;\left(\left(b^{(k)}\right):\left(\phi^{(k)}\right)\right)$
$\left[(c): \psi^{(1)}, \ldots, \psi^{(k)}\right]:\left[\left(d^{(1)}\right):\left(\delta^{(1)}\right)\right] ; \ldots ;\left[\left(d^{(k)}\right):\left(\delta^{(k)}\right)\right]$

$z_{1}, \ldots z_{k}$ 


$$
\begin{aligned}
= & \frac{1}{(2 \pi \omega)^{k}} \int_{-\omega \infty}^{\omega \infty} \ldots \int_{-\omega \infty}^{\omega \infty} \frac{\prod_{j=1}^{\lambda} \Gamma\left(1-a_{j}+\sum_{i=1}^{k} \theta_{j}^{(i)} s_{i}\right)}{\prod_{j=\lambda+1}^{A} \Gamma\left(a_{j}-\sum_{i=1}^{k} \theta_{j}^{(i)} s_{i}\right) \prod_{j=1}^{C} \Gamma\left(1-c_{j}+\sum_{i=1}^{k} \psi{ }_{j}^{(i)} s_{i}\right)} \\
\times & \frac{\prod_{j=1}^{\mu^{(1)}} \Gamma\left(1-d_{j}+\delta_{j}^{(1)} s_{1}\right) \ldots \prod_{j=1}^{\mu^{(k)}} \Gamma\left(1-d_{j}+\delta_{j}^{(k)} s_{k}\right)}{\prod_{j=\mu^{(1)}+1}^{D^{(1)}} \Gamma\left(d_{j}-\delta_{j}^{(1)} s_{1}\right) \ldots \prod_{j=\mu^{(k)}+1}^{D^{(k)}} \Gamma\left(d_{j}-\delta_{j}^{(k)} s_{k}\right)} \\
& \prod_{j=1}^{v^{(1)}} \Gamma\left(1-b_{j}+\phi_{j}^{(1)} s_{1}\right) \ldots \prod_{j=1}^{v^{(k)}} \Gamma\left(1-b_{j}+\phi_{j}^{(k)} s_{k}\right) \\
\times & \prod_{j=v^{(1)}+1}^{B^{(1)}} \Gamma\left(b_{j}-\phi_{j}^{(1)} s_{1}\right) \ldots \prod_{j=v^{(k)}+1}^{B^{(k)}} \Gamma\left(b_{j}-\phi_{j}^{(k)} s_{k}\right)
\end{aligned}
$$

The integral in equation (1.25) converges absolutely if

$$
\left|\arg \left(z_{i}\right)\right|<\frac{\pi}{2} \Delta_{i}
$$

where,

$$
\begin{aligned}
& \Delta_{i}=\sum_{j=1}^{\lambda} \theta_{j}^{(i)}-\sum_{j=\lambda+1}^{A} \theta_{j}^{(i)}+\sum_{j=1}^{v^{(i)}} \phi_{j}^{(i)}-\sum_{j=v^{(i)}+1}^{B^{(i)}} \phi_{j}^{(i)}+\sum_{j=1}^{\mu^{(i)}} \delta_{j}^{(i)} \\
& -\sum_{j=\mu^{(i)}+1}^{D^{(i)}} \delta_{j}^{(i)}-\sum_{j=1}^{C} \psi_{j}^{(i)}>0, i \in\{1, \ldots, k\} .
\end{aligned}
$$

If in the equations (1.25)-(1.27) all $\theta^{s}, \phi^{s}, \delta^{s}, a n d \psi^{s}$ are unity, then the integral in equation (1.25) becomes generalized G-function for the prescribed conditions given in the equations (1.26) and (1.27).

\section{Results}

In this section, we obtain following identities for above generalized fractional integral operators defined in the equations (1.19) and (1.20) when $g\{z\}=(1-z)^{-\lambda}, \lambda \in C, \operatorname{Re}(\lambda)>0$.

\section{Theorem 2.1:}

For the given conditions and the definitions in the equations (1.6), (1.12)-(1.14), (1.18) and (1.19) and $g\{z\}=(1-z)^{-\lambda}, \lambda \in C, \operatorname{Re}(\lambda)>0$, there exists an identity 


$$
\begin{aligned}
& H^{\alpha, b, c}\left\{(1-z)^{-\lambda}, \Psi\left(z_{1}, \ldots, z_{k}\right)\right\}\left(x_{1}, \ldots, x_{k}\right)=\sum_{r_{1}, \ldots, r_{k}=0}^{\infty} A_{r_{1}, \ldots, r_{k}}\left(c_{1}\right)_{r_{1}} \ldots\left(c_{k}\right)_{r_{k}} \\
& \times \alpha_{1}^{r_{1}} \ldots \alpha_{k}^{r_{k}} \alpha_{k+1}^{r_{k+1}} \ldots \alpha_{l}^{r_{l}} \sum_{m_{1}, \ldots, m_{k}=0}^{\infty} \frac{(\lambda)_{m_{1}+\ldots+m_{k}}\left(b_{1}\right)_{m_{1}} \ldots\left(b_{k}\right)_{m_{k}}}{\left(b_{1}+\ldots+b_{k}\right)_{m_{1}+\ldots+m_{k}} m_{1} ! \ldots m_{k} !} E_{z_{1}}^{c_{1}+r_{1}, b_{1}-c_{1}+m_{1}-r_{1}-1} \ldots \\
& . . E_{z_{k}}^{c_{k}+r_{k}, b_{k}-c_{k}+m_{k}-r_{k}-1}\left\{\Psi\left(z_{1}, \ldots, z_{k}\right)\right\}\left(x_{1}, \ldots, x_{k}\right)
\end{aligned}
$$

provided that $\operatorname{Re}\left(b_{i}-c_{i}\right)>0,\left(m_{i}-r_{i}\right) \geq 0,\left(m_{i} \in\{0,1,2, \ldots\}\right)$ and $\left(r_{i} \in\{0,1,2, \ldots\}\right), \forall i=1,2, \ldots, k$.

Proof: Consider the operation of the function $\Psi\left(z_{1}, \ldots, z_{k}\right) \in\{(0, \infty)\}^{k}$ due to the generalized fractional integral operator defined in equation (1.19) for $g\{z\}=(1-z)^{-\lambda}$,

$\lambda \in C, \operatorname{Re}(\lambda)>0$ and then express $\Phi\left(\alpha_{1}, \ldots, \alpha_{l}\right)$ defined by equation (1.18) and $F\{g\}(b ; z, x)$ due to equations (1.12)-(1.14), and then in the inner integral set $v_{i}=\frac{u_{i} x_{i}}{z_{i}}, \forall i=1,2, \ldots, k$ and on solving it by well known Dirichlet integral formula we get left hand side of equation (2.1) in the form:

$$
\begin{aligned}
& \sum_{r_{1}, \ldots, r_{l}=0}^{\infty} A_{r_{1}, \ldots, r_{l}} \alpha_{1}^{r_{1}} \ldots \alpha_{l}^{r_{l}} \sum_{m_{1}, \ldots, m_{k}=0}^{\infty} \frac{(\lambda)_{m_{1}+\ldots+m_{k}}\left(b_{1}\right)_{m_{1}} \ldots\left(b_{k}\right)_{m_{k}}}{\left(b_{1}+\ldots+b_{k}\right)_{m_{1}+\ldots+m_{k}} m_{1} ! \ldots m_{k} !} \frac{\left(x_{1}^{-1}\right)^{b_{1}+m_{1}-1}}{\Gamma\left(c_{1}\right)} \ldots \frac{\left(x_{k}^{-1}\right)^{b_{k}+m_{k}-1}}{\Gamma\left(c_{k}\right)} \\
& \times \int_{0}^{x_{1}} \ldots \int_{0}^{x_{k}}\left(x_{1}-z_{1}\right)^{c_{1}+r_{1}-1} \ldots\left(x_{k}-z_{k}\right)^{c_{k}+r_{k}-1} z_{1}^{b_{1}+m_{1}-r_{1}-c_{1}-1} \ldots z_{k}^{b_{k}+m_{k}-r_{k}-c_{k}-1} \Psi\left(z_{1}, \ldots, z_{k}\right) d z_{1} \ldots d z_{k}
\end{aligned}
$$

Now, make an appeal to the equations (1.6) and (2.2) we immediately find right hand side of equation (2.1).

Theorem 2.2: For the given conditions and the definitions in equations (1.8), (1.15)-(1.17), (1.18) and (1.20) and there exists an identity

$$
\begin{aligned}
& P^{\alpha, b, c}\left\{(1-z)^{-\lambda}, \Psi\left(z_{1}, \ldots, z_{k}\right)\right\}\left(x_{1}, \ldots, x_{k}\right)=(-1)^{k-1} \sum_{r_{1}, \ldots, r_{k}=0}^{\infty} A_{r_{1}, \ldots, r_{k}}\left(c_{1}\right)_{r_{1}} \ldots\left(c_{k}\right)_{r_{k}} \\
& \times \alpha_{1}^{r_{1}} \ldots \alpha_{k}^{r_{k}} \alpha_{k+1}^{r_{k+1}} \ldots \alpha_{l}^{r_{l}} \sum_{m_{1}, \ldots, m_{k}=0}^{\infty} \frac{(\lambda)_{m_{1}+\ldots+m_{k}}\left(b_{1}\right)_{m_{1}} \ldots\left(b_{k}\right)_{m_{k}}}{\left(b_{1}+\ldots+b_{k}\right)_{m_{1}+\ldots+m_{k}} m_{1} ! \ldots m_{k} !} K_{z_{1}}^{c_{1}+r_{1}, b_{1}-c_{1}+m_{1}-r_{1}} \ldots \\
& . . K_{z_{k}}^{c_{k}+r_{k}, b_{k}-c_{k}+m_{k}-r_{k}}\left\{\Psi\left(z_{1}, \ldots, z_{k}\right)\right\}\left(x_{1}, \ldots, x_{k}\right) \\
& \text { provided that } \operatorname{Re}\left(b_{i}-c_{i}\right)>0,\left(m_{i}-r_{i}\right) \geq 0,\left(m_{i} \in\{0,1,2, \ldots\}\right) \text { and }\left(r_{i} \in\{0,1,2, \ldots\}\right), \forall i=1,2, \ldots, k .
\end{aligned}
$$

Proof:

Operate the function $\Psi\left(z_{1}, \ldots, z_{k}\right) \in\{(0, \infty)\}^{k}$ due to the generalized fractional integral operator defined in equation (1.20) for $g\{z\}=(1-z)^{-\lambda}, \lambda \in C, \operatorname{Re}(\lambda)>0$, and then express $\Phi\left(\alpha_{1}, \ldots, \alpha_{l}\right)$ defined by the equation (1.18) and $F *\{g\}(b ; z, x)$ due to equations (1.15)-(1.17), and then in the inner integral set $v_{i}=\frac{z_{i}}{u_{i} x_{i}}, \forall i=1,2, \ldots, k$ and on solving it by well known Dirichlet integral formula we get left hand side of the equation (2.3) in the form: 


$$
\begin{aligned}
& (-1)^{k-1} \sum_{r_{1}, \ldots, r_{l}=0}^{\infty} A_{r_{1}, \ldots, r_{l}} \alpha_{1}^{r_{1}} \ldots \alpha_{l}^{r_{l}} \sum_{m_{1}, \ldots, m_{k}=0}^{\infty} \frac{(\lambda)_{m_{1}+\ldots+m_{k}}\left(b_{1}\right)_{m_{1}} \ldots\left(b_{k}\right)_{m_{k}}}{\left(b_{1}+\ldots+b_{k}\right)_{m_{1}+\ldots+m_{k}} m_{1} ! \ldots m_{k} !} \frac{\left(x_{1}\right)^{b_{1}+m_{1}-r_{1}-c_{1}}}{\Gamma\left(c_{1}\right)} \ldots \frac{\left(x_{k}\right)^{b_{k}+m_{k}-r_{k}-c_{k}}}{\Gamma\left(c_{k}\right)} \\
& \times \int_{x_{1}}^{\infty} \ldots \int_{x_{k}}^{\infty}\left(z_{1}-x_{1}\right)^{c_{1}+r_{1}-1} \ldots\left(z_{k}-x_{k}\right)^{c_{k}+r_{k}-1} z_{1}^{-b_{1}-m_{1}} \ldots z_{k}^{-b_{k}-m_{k}} \Psi\left(z_{1}, \ldots, z_{k}\right) d z_{1} \ldots d z_{k}
\end{aligned}
$$

Now, make an appeal to the equation (1.8) and (2.4) we immediately find right hand side of the (2.3).

\section{Theorem 2.3:}

For the given conditions and the definitions given in the Theorem A and Theorem B, the images of the unity due to operators given in the equations (1.19) and (1.20) consist of the following identical relation

$$
\begin{aligned}
& H^{\alpha, b, c}\left\{(1-z)^{-\lambda}, 1\right\}\left(x_{1}, \ldots, x_{k}\right)=(-1)^{k-1} P^{\alpha, b, c}\left\{(1-z)^{-\lambda}, 1\right\}\left(x_{1}, \ldots, x_{k}\right) \\
& =\frac{\Gamma\left(b_{1}-c_{1}\right) \ldots \Gamma\left(b_{k}-c_{k}\right) \Gamma\left(c_{1}+\ldots+c_{k}-\lambda\right)}{B(b) \Gamma\left(b_{1}+\ldots+b_{k}-\lambda\right) \Gamma\left(c_{1}+\ldots+c_{k}\right)} \sum_{r_{1}, \ldots, r_{k}=0}^{\infty} A_{r_{1}, \ldots, r_{k}}\left(c_{1}\right)_{r_{1}} \ldots\left(c_{k}\right)_{r_{k}} \\
& \times \frac{\left(c_{1}+\ldots+c_{k}-\lambda\right)_{r_{1}+\ldots+r_{k}}(-1)^{r_{1}} \ldots(-1)^{r_{k}}}{\left(1-b_{1}+c_{1}\right)_{r_{1}} \ldots\left(1-b_{k}+c_{k}\right)_{r_{k}}\left(c_{1}+\ldots+c_{k}\right)_{r_{1}+\ldots+r_{k}}} \alpha_{1}^{r_{1}} \ldots \alpha_{k}^{r_{k}} \alpha_{k+1}^{r_{k+1} \ldots \alpha_{l}^{r_{l}}}
\end{aligned}
$$

provided that $\operatorname{Re}\left(\mathrm{b}_{\mathrm{i}}\right)>0, i=1,2, \ldots, k$ and $\operatorname{Re}(\lambda)<\operatorname{Re}\left(b_{1}+\ldots+b_{k}\right)$

and $\operatorname{Re}\left(b_{i}-c_{i}\right)>0,\left(m_{i}-r_{i}\right) \geq 0,\left(m_{i} \in\{0,1,2, \ldots\}\right)$ and $\left(r_{i} \in\{0,1,2, \ldots\}\right), \forall i=1,2, \ldots, k$.

Proof: In the equations (2.1) and (2.3) set $\Psi\left(z_{1}, \ldots, z_{k}\right)$ then on solving these we find the relation given in the equation (2.5).

\section{Applications}

In this section, we make an application of the operators given in the equation (1.19) and (1.20), for $g\{z\}=(1-z)^{-\lambda}, \lambda \in C, \operatorname{Re}(\lambda)>0$, and then evaluate the images of the general class of multivariable polynomials (Srivastava and Garg, 1987) with products of the exponential functions.

The general class of multivariable polynomials is defined by (Srivastava and Garg, 1987)

$$
S_{L}^{h_{1}, \ldots, h_{m}}\left(x_{1}, \ldots, x_{m}\right)=\sum_{s_{1}, \ldots, s_{m}=0}^{h_{1} s_{1}+\ldots+h_{m} s_{m} \leq L}(-L)_{h_{1} s_{1}+\ldots+h_{m} s_{m}} A\left[L ; s_{1}, \ldots, s_{m}\right] \frac{x_{1}^{s_{1}}}{s_{1} !} \ldots \frac{x_{m}^{s_{m}}}{s_{m} !}
$$

where, $\mathrm{h}_{1}, \ldots, \mathrm{h}_{\mathrm{m}}$ are arbitrary positive integers and the coefficients $A\left[L ; s_{1}, \ldots, s_{m}\right],\left(L, s_{j} \in N_{0}=\{0,1,2, \ldots\}, j=1, \ldots, m\right)$ are arbitrary constants real or complex.

For $\mathrm{m}=1$, of the polynomials defined by the equation (3.1) would correspond to polynomials (Srivastava, 1972 )

$S_{L}^{h}(x)=\sum_{s=0}^{\left[\frac{L}{h}\right]}(-L)_{h s} A_{L, s} \frac{x^{s}}{s !}$

where, $\mathrm{h}$ is arbitrary positive integer and the coefficients, $A_{L, s}\left(L, s \in N_{0}=\{0,1,2, \ldots\}\right)$ are arbitrary constants real or complex .

\section{Theorem 3.1:}

For the conditions and the definitions given in the Theorem A, the image of the distribution 


$$
\begin{aligned}
& \Psi\left(z_{1}, \ldots z_{k}\right)=\exp \left[-\beta_{1} z_{1}^{-1}\left(x_{1}-z_{1}\right)\right] \ldots \exp \left[-\beta_{k} z_{k}^{-1}\left(x_{k}-z_{k}\right)\right] \\
& \times S_{L}^{h_{1}, \ldots, h_{m}}\left(\gamma_{1} z_{1}^{-1}\left(x_{1}-z_{1}\right), \ldots, \gamma_{k} z_{k}^{-1}\left(x_{k}-z_{k}\right), \gamma_{k+1}, \ldots, \gamma_{m}\right), \forall z_{i} \in\left(0, x_{i}\right), x_{i}>0, i \in\{1, \ldots, k\}, \\
& \text { and } \Psi\left(z_{1}, \ldots, z_{k}\right)=0, \text { otherwise }
\end{aligned}
$$

exists and there holds the formula

$$
\begin{aligned}
& H^{\alpha, b, c}\left\{(1-z)^{-\lambda}, \exp \left[-\beta_{1} z_{1}^{-1}\left(x_{1}-z_{1}\right)\right] \ldots \exp \left[-\beta_{k} z_{k}^{-1}\left(x_{k}-z_{k}\right)\right]\right. \\
& \left.\times S_{L}^{h_{1}, \ldots, h_{m}}\left(\gamma_{1} z_{1}^{-1}\left(x_{1}-z_{1}\right), \ldots, \gamma_{k} z_{k}^{-1}\left(x_{k}-z_{k}\right), \gamma_{k+1}, \ldots, \gamma_{m}\right)\right\}\left(x_{1}, \ldots, x_{k}\right) \\
& =\frac{1}{B(b) \Gamma\left(b_{1}+\ldots+b_{k}-\lambda\right) \Gamma\left(c_{1}\right) \ldots \Gamma\left(c_{k}\right)} \sum_{s_{1}, \ldots, s_{m}=0}^{h_{1} s_{1}+\ldots+h_{m} s_{m} \leq L}(-L)_{h_{1} s_{1}+\ldots+h_{m} s_{m}} A\left[L ; s_{1}, \ldots, s_{m}\right] \frac{\gamma_{1}^{s_{1}}}{s_{1} !} \ldots \frac{\gamma_{m}^{s_{m}}}{s_{m} !} \\
& \times \sum_{r_{1}, \ldots r_{l}=0}^{\infty} A_{r_{1}, \ldots, r_{l}} \alpha_{1}^{r_{1}} \ldots \alpha_{l}^{r_{l}} G \begin{array}{c}
0,1:(2,1) ; \ldots ;(2,1)\left(\left[1+\lambda-c_{1}-r_{1}-s_{1}-\ldots-c_{k}-r_{k}-s_{k}: 1, \ldots, 1\right]\right. \\
1,1:[1,2] ; \ldots ;[1,2]
\end{array} \\
& :\left[1-c_{1}-r_{1}-s_{1}: 1\right] ; \ldots ;\left[1-c_{1}-r_{1}-s_{1}-\ldots-c_{k}-r_{k}-s_{k}: 1, \ldots, 1\right] \\
& \left.:[0: 1],\left[b_{1}-c_{1}-r_{1}-s_{1}: 1\right] ; \ldots ;[0: 1],\left[b_{k}-c_{k}-r_{k}-s_{k}: 1\right] ; \beta_{1}, \ldots, \beta_{k}\right)
\end{aligned}
$$

provided that

$$
\left|\arg \left(\beta_{i}\right)\right|<\frac{3 \pi}{2}, \operatorname{Re}\left(c_{i}\right)<\operatorname{Re}\left(b_{i}\right), \operatorname{Re}\left(c_{i}\right)>0, \forall i=1,2, \ldots, k, \operatorname{Re}(\lambda)<\operatorname{Re}\left(c_{1}+\ldots+c_{k}\right)
$$

and all conditions of theorem 2.1 and of equation (3.1) are followed.

Proof: In the left hand side of equation (3.4), we proceed the actions taken in the theorem 2.1, so that we express the distribution $\Psi\left(z_{1}, \ldots, z_{k}\right)$ with the help of equation (3.3) in the equation (2.2), then define the general class of multivariable polynomials due to equation (3.1) and then in it apply the contour integral formula of exponential functions (Mathai and Saxena, 1973) such that

$$
e^{-x}=\frac{1}{2 \pi \omega} \int_{-\infty \infty}^{\infty \infty} \Gamma(-\xi)(x)^{\xi} d \xi,|x|<\infty, \omega=\sqrt{(-1)}
$$

Then after changing the order of integration and the summation and then on solving it we find that

$$
\begin{aligned}
& \frac{1}{\Gamma\left(b_{1}\right) \ldots \Gamma\left(b_{k}\right) \Gamma\left(c_{1}\right) \ldots \Gamma\left(c_{k}\right)} \sum_{s_{1}, \ldots, s_{m}=0}^{h_{1} s_{1}+\ldots+h_{m} s_{m} \leq L}(-L)_{h_{1} s_{1}+\ldots+h_{m} s_{m}} A\left[L ; s_{1}, \ldots, s_{m}\right] \frac{\gamma_{1}^{s_{1}}}{s_{1} !} \ldots \frac{\gamma_{m}^{s_{m}}}{s_{m} !} \\
& \times \sum_{r_{1}, \ldots r_{l}=0}^{\infty} A_{r_{1}, \ldots, r_{l}} \alpha_{1}^{r_{1}} \ldots \alpha_{l}^{r_{l}} \frac{1}{(2 \pi \omega)^{k}} \int_{-\omega \infty}^{\omega \infty} \ldots \int_{-\omega \infty}^{\infty \infty 0} \Gamma\left(-\xi_{1}\right) \ldots \Gamma\left(-\xi_{k}\right) \Gamma\left(c_{1}+r_{1}+s_{1}+\xi_{1}\right) \Gamma\left(b_{1}-c_{1}-r_{1}-s_{1}-\xi_{1}\right) \\
& \ldots \Gamma\left(c_{k}+r_{k}+s_{k}+\xi_{k}\right) \Gamma\left(b_{k}-c_{k}-r_{k}-s_{k}-\xi_{k}\right) \beta_{1}^{\xi_{1}} \ldots \beta_{k}^{\xi_{k}} \\
& \times \sum_{m_{1}, \ldots m_{k}=0}^{\infty} \frac{(\lambda)_{m_{1}+\ldots+m_{k}}\left(b_{1}-c_{1}-r_{1}-s_{1}-\xi_{1}\right)_{m_{1}} \ldots\left(b_{k}-c_{k}-r_{k}-s_{k}-\xi_{k}\right)_{m_{k}}}{\left(b_{1}+\ldots+b_{k}\right)_{m_{1}+\ldots+m_{k}} m_{1} ! \ldots m_{k} !} d \xi_{1} \ldots d \xi_{k}
\end{aligned}
$$

Now, in the inner series in equation (3.6), using the formula (Lauricella, 1893, p.150) (See also, Appell and Kampe' de Fe'riet, 1926, p.117 and Srivastava and Manocha , 1984, eq.(8) ) such that 


$$
\begin{aligned}
& F_{D}^{(k)}\left[a, b_{1}, \ldots, b_{k} ; c ; 1, \ldots, 1\right]=\sum_{m_{1}, \ldots, m_{k}=0}^{\infty} \frac{(a)_{m_{1}+\ldots+m_{k}}\left(b_{1}\right)_{m_{1}} \ldots\left(b_{k}\right)_{m_{k}}}{(c)_{m_{1}+\ldots+m_{k}} m_{1} ! \ldots m_{k} !} \\
& =\frac{\Gamma(c) \Gamma\left(c-a-b_{1}-\ldots-b_{k}\right)}{\Gamma(c-a) \Gamma\left(c-b_{1}-\ldots-b_{k}\right)}, c \neq 0,-1,-2, \ldots, \text { and } \operatorname{Re}\left(c-a-b_{1}-\ldots-b_{k}\right)>0,
\end{aligned}
$$

and then defining the multivariable G-function as making an application of the equations (1.25)-(1.27), we evaluate right hand side of equation (3.4).

In the same manner, we state and prove following theorem:

\section{Theorem 3.2:}

For the conditions and the definitions given in the Theorem $\mathrm{B}$, the image of the distribution

$$
\begin{aligned}
& \Psi\left(z_{1}, \ldots z_{k}\right)=\exp \left[-\beta_{1}\left(z_{1}-x_{1}\right)\right] \ldots \exp \left[-\beta_{k}\left(z_{k}-x_{k}\right)\right] \\
& \times S_{L}^{h_{1}, \ldots, h_{m}}\left(\gamma_{1}\left(z_{1}-x_{1}\right), \ldots, \gamma_{k}\left(z_{k}-x_{k}\right), \gamma_{k+1}, \ldots, \gamma_{m}\right), \forall z_{i} \in\left(x_{i}, \infty\right), x_{i}>0, i \in\{1, \ldots, k\},
\end{aligned}
$$

and $\Psi\left(z_{1}, \ldots, z_{k}\right)=0$, otherwise

exists and there holds the formula

$$
\begin{aligned}
& p^{\alpha, b, c}\left\{(1-z)^{-\lambda}, \exp \left[-\beta_{1}\left(z_{1}-x_{1}\right)\right] \ldots \exp \left[-\beta_{k}\left(z_{k}-x_{k}\right)\right]\right. \\
& \left.\times S_{L}^{h_{1}, \ldots, h_{m}}\left(\gamma_{1}\left(z_{1}-x_{1}\right), \ldots, \gamma_{k}\left(z_{k}-x_{k}\right), \gamma_{k+1}, \ldots, \gamma_{m}\right)\right\}\left(x_{1}, \ldots, x_{k}\right) \\
& =\frac{(-1)^{k-1}}{B(b) \Gamma\left(b_{1}+\ldots+b_{k}-\lambda\right) \Gamma\left(c_{1}\right) \ldots \Gamma\left(c_{k}\right)} \sum_{s_{1}, \ldots, s_{m}=0}^{h_{1} s_{1}+\ldots+h_{m} s_{m} \leq L}(-L)_{h_{1} s_{1}+\ldots+h_{m} s_{m}} A\left[L ; s_{1}, \ldots, s_{m}\right] \frac{\left(\gamma_{1} x_{1}\right)^{s_{1}}}{s_{1} !} \ldots \frac{\left(\gamma_{k} x_{k}\right)^{s_{k}}}{s_{k} !} \frac{\gamma_{k+1}^{s_{k+1}}}{s_{k+1} !} \ldots \frac{\gamma_{m}^{s_{m}}}{s_{m} !} \\
& \times \sum_{r_{1}, \ldots r_{l}=0}^{\infty} A_{r_{1}, \ldots, r_{l}} \alpha_{1}^{r_{1}} \ldots \alpha_{l}^{r_{l}} G \quad 0,1:(2,1) ; \ldots ;(2,1)\left(\left[1+\lambda-c_{1}-r_{1}-s_{1}-\ldots-c_{k}-r_{k}-s_{k}: 1, \ldots, 1\right]\right. \\
& 1,1:[1,2] ; \ldots ;[1,2]\left(\left[1-c_{1}-r_{1}-s_{1}-\ldots-c_{k}-r_{k}-s_{k}: 1, \ldots, 1\right]\right. \\
& :\left[1-c_{1}-r_{1}-s_{1}: 1\right] ; \ldots ;\left[1-c_{k}-r_{k}-s_{k}: 1\right] ; \\
& \left.:[0: 1],\left[b_{1}-c_{1}-r_{1}-s_{1}: 1\right] ; \ldots ;[0: 1],\left[b_{k}-c_{k}-r_{k}-s_{k}: 1\right] ; \beta_{1} x_{1}, \ldots, \beta_{k} x_{k}\right)
\end{aligned}
$$

provided that

$$
\left|\arg \left(\beta_{i}\right)\right|<\frac{3 \pi}{2}, \operatorname{Re}\left(c_{i}\right)<\operatorname{Re}\left(b_{i}\right), \operatorname{Re}\left(c_{i}\right)>0, \forall i=1,2, \ldots, k, \operatorname{Re}(\lambda)<\operatorname{Re}\left(c_{1}+\ldots+c_{k}\right)
$$

and all conditions of theorem 2.2 and of equation (3.1) are followed.

With the help of the identities derived in the equations (2.1) and (3.4), we may evaluate the images of the general class of multivariable polynomials with products of the exponential functions in terms of the multiple series consisting general coefficients and the multivariable G-function. In the similar manner, from the identities derived in the equations (2.3) and (3.9), we may again evaluate other images of the general class of multivariable polynomials with products of the exponential functions in terms of the multiple series consisting general coefficients and the multivariable G-function.

\section{Deductions}

The Dirichlet average for $x^{n}$ has been defined in the form (Carlson, 1977, p.91)

$$
R_{n}\left(b, b^{\prime} ; x, y\right)=\frac{\Gamma\left(b+b^{\prime}\right)}{\Gamma(b) \Gamma\left(b^{\prime}\right)} \int_{0}^{1}[x u+y(1-u)]^{n} u^{b-1}(1-u)^{b^{\prime}-1} d u
$$




\section{Corollary 4.1}

For the generalized fractional integral operator given in equation (1.19) and the Dirichet average defined in equation (4.1) and for $g\{z\}=(1-z)^{-\lambda}, \lambda \in C, \operatorname{Re}(\lambda)>0$, and for $1=\mathrm{k}=2$, following relation exists

$$
\begin{aligned}
& H^{\alpha, \alpha^{\prime}, b, b^{\prime}, c, c^{\prime}}\left\{(1-z)^{-\lambda}, \Psi\left(z, z^{\prime}\right)\right\}\left(x, x^{\prime}\right)=\frac{(x)^{1-b}\left(x^{\prime}\right)^{1-b^{\prime}}}{\Gamma(c) \Gamma\left(c^{\prime}\right)} \sum_{n=0}^{\infty} \frac{(\lambda)_{n}}{n !} \int_{0}^{x} \int_{0}^{x^{\prime}}(x-z)^{c-1}\left(x^{\prime}-z^{\prime}\right)^{c^{\prime}-1} \\
& \times(z)^{b-c-1}\left(z^{\prime}\right)^{b^{\prime}-c^{\prime}-1} R_{n}\left(b, b^{\prime} ; z(x)^{-1}, z^{\prime}\left(x^{\prime}\right)^{-1}\right) \Phi\left(\alpha(z)^{-1}(x-z), \alpha^{\prime}\left(z^{\prime}\right)^{-1}\left(x^{\prime}-z^{\prime}\right)\right) \Psi\left(z, z^{\prime}\right) d z d z^{\prime}
\end{aligned}
$$

\section{Corollary 4.2}

For the generalized fractional integral operator given in the equation (1.20) and the Dirichet average defined in equation (4.1) and for $g\{z\}=(1-z)^{-\lambda}, \lambda \in C, \operatorname{Re}(\lambda)>0$, and for $\mathrm{l}=\mathrm{k}=2$, following relation exists

$$
\begin{aligned}
& R^{\alpha, \alpha^{\prime}, b, b^{\prime}, c, c^{\prime}}\left\{(1-z)^{-\lambda}, \Psi\left(z, z^{\prime}\right)\right\}\left(x, x^{\prime}\right)=\frac{(x)^{b-c}\left(x^{\prime}\right)^{b^{\prime}-c^{\prime \prime}}}{\Gamma(c) \Gamma\left(c^{\prime}\right)} \sum_{n=0}^{\infty} \frac{(\lambda)_{n}}{n !} \int_{x}^{\infty} \int_{x^{\prime}}^{\infty}(z-x)^{c-1}\left(z^{\prime}-x^{\prime}\right)^{c^{\prime}-1} \\
& \times(z)^{-b}\left(z^{\prime}\right)^{-b^{\prime}} R_{n}\left(b, b^{\prime} ; x(z)^{-1}, x^{\prime}\left(z^{\prime}\right)^{-1}\right) \Phi\left(\alpha(x)^{-1}(z-x), \alpha^{\prime}\left(x^{\prime}\right)^{-1}\left(z^{\prime}-x^{\prime}\right)\right) \Psi\left(z, z^{\prime}\right) d z d z^{\prime}
\end{aligned}
$$

Further making an appeal to the equations (2.1) and (4.2) for $g\{z\}=(1-z)^{-\lambda}, \lambda \in C, \operatorname{Re}(\lambda)>0$, and for $1=\mathrm{k}=2$, following relation exists

$$
\begin{aligned}
& \frac{(x)^{1-b}\left(x^{\prime}\right)^{1-b^{\prime}}}{\Gamma(c) \Gamma\left(c^{\prime}\right)} \sum_{n=0}^{\infty} \frac{(\lambda)_{n}}{n !} \int_{0}^{x} \int_{0}^{x^{\prime}}(x-z)^{c-1}\left(x^{\prime}-z^{\prime}\right)^{c^{\prime}-1} \\
& \times(z)^{b-c-1}\left(z^{\prime}\right)^{b^{\prime}-c^{\prime}-1} R_{n}\left(b, b^{\prime} ; z(x)^{-1}, z^{\prime}\left(x^{\prime}\right)^{-1}\right) \Phi\left(\alpha(z)^{-1}(x-z), \alpha^{\prime}\left(z^{\prime}\right)^{-1}\left(x^{\prime}-z^{\prime}\right)\right) \Psi\left(z, z^{\prime}\right) d z d z^{\prime} \\
& =\sum_{r, r^{\prime}=0}^{\infty} A_{r, r^{\prime}}(c)_{r}\left(c^{\prime}\right)_{r^{\prime}}(\alpha)^{r}\left(\alpha^{\prime}\right)^{r^{\prime}} \sum_{m, m^{\prime}=0}^{\infty} \frac{(\lambda)_{m+m^{\prime}}(b)_{m}\left(b^{\prime}\right)_{m^{\prime}} E_{z}^{c+r, b-c+m-r-1} E_{z^{\prime}}^{c^{\prime}+r^{\prime}, b^{\prime}-c^{\prime}+m^{\prime}-r^{\prime}-1}\left\{\Psi\left(z, z_{m+m^{\prime}} m ! m^{\prime}\right)\right\}\left(x, x^{\prime}\right)}{\left(b+b^{\prime}\right)}
\end{aligned}
$$

provided that $\operatorname{Re}(b-c)>0, \operatorname{Re}\left(b^{\prime}-c^{\prime}\right)>0,(m-r) \geq 0,\left(m^{\prime}-r^{\prime}\right) \geq 0,\left(m, m^{\prime} \in\{0,1,2, \ldots\}\right)$,

$\left(r, r^{\prime} \in\{0,1,2, \ldots\}\right)$.

Again making an appeal to equations (2.3) and (4.3) for $g\{z\}=(1-z)^{-\lambda}, \lambda \in C, \operatorname{Re}(\lambda)>0$, and for $1=\mathrm{k}=2$ we find another identity

$$
\begin{aligned}
& \frac{(x)^{b-c}\left(x^{\prime}\right)^{b^{\prime}-c^{\prime \prime}}}{\Gamma(c) \Gamma\left(c^{\prime}\right)} \sum_{n=0}^{\infty} \frac{(\lambda)_{n}}{n !} \int_{x}^{\infty} \int_{x^{\prime}}^{\infty}(z-x)^{c-1}\left(z^{\prime}-x^{\prime}\right)^{c^{\prime}-1} \\
& \times(z)^{-b}\left(z^{\prime}\right)^{-b^{\prime}} R_{n}\left(b, b^{\prime} ; x(z)^{-1}, x^{\prime}\left(z^{\prime}\right)^{-1}\right) \Phi\left(\alpha(x)^{-1}(z-x), \alpha^{\prime}\left(x^{\prime}\right)^{-1}\left(z^{\prime}-x^{\prime}\right)\right) \Psi\left(z, z^{\prime}\right) d z d z^{\prime} \\
& =(-1)^{k-1} \sum_{r, r^{\prime}=0}^{\infty} A_{r, r^{\prime}}(c)_{r}\left(c^{\prime}\right)_{r^{\prime}}(\alpha)^{r}\left(\alpha^{\prime}\right)^{r^{\prime}} \sum_{m, m^{\prime}=0}^{\infty} \frac{(\lambda)_{m+m^{\prime}}(b)_{m}\left(b^{\prime}\right)_{m^{\prime}} K_{z}^{c+r, b-c+m-r} K_{z^{\prime}}^{c^{\prime}+r^{\prime}, b^{\prime}-c^{\prime}+m^{\prime}-r^{\prime}}\left\{\Psi\left(z, z^{\prime}\right)\right\}\left(x, x_{m+m^{\prime}} m ! m^{\prime} !\right.}{\left(b+b^{\prime}\right)}
\end{aligned}
$$

$$
\text { provided that } \operatorname{Re}(b-c)>0, \operatorname{Re}\left(b^{\prime}-c^{\prime}\right)>0,(m-r) \geq 0,\left(m^{\prime}-r^{\prime}\right) \geq 0,\left(m, m^{\prime} \in\{0,1,2, \ldots\}\right),
$$

$\left(r, r^{\prime} \in\{0,1,2, \ldots\}\right)$.

If we express the function $\Psi\left(z, z^{\prime}\right)$, in according to the section 3 in equations (4.4) and (4.5) we may find its images in form of the series involving two variables G-function. Again these results having general coefficients so that on specializing them we may 
obtain the images of several hypergeometric functions and the polynomials scattered in the literature with products of exponential functions in form of the series consisting two variables G-function.

\section{Application in Statistics}

Theorem 5.1 suppose that $\left(u_{1}, \ldots, u_{k}\right)$ is a $\mathrm{k}$-dimensional random variable independent to the variable $\left(z_{1}, \ldots, z_{k}\right)$ with density $f\left(u_{1}, \ldots, u_{k}\right)$ given by

$$
f\left(u_{1}, \ldots, u_{k}\right)=\frac{z_{k}^{b_{k}} x_{k}^{-b_{k}} u_{1}^{b_{1}-1} \ldots u_{k-1}^{b_{k-1}-1}}{B(b) K\left(x_{1}, \ldots, x_{k} ; z_{1}, \ldots, z_{k}\right)}\left(1-\frac{u_{1} x_{1}}{z_{1}}-\ldots-\frac{u_{k-1} x_{k-1}}{z_{k-1}}\right)^{b_{k}-1}
$$

$0 \leq u_{1} \leq \frac{z_{1}}{x_{1}} ; x_{1}>z_{1}>0, \ldots, 0 \leq u_{k-1} \leq \frac{z_{k-1}}{x_{k-1}} ; x_{k-1}>z_{k-1}>0, \frac{u_{k} x_{k}}{z_{k}}=1-\frac{u_{1} x_{1}}{z_{1}}-\ldots-\frac{u_{k-1} x_{k-1}}{z_{k-1}} ;$

$x_{k}>z_{k}>0,\left(z_{1}, \ldots, z_{k}\right) \in R^{k}$, the constants $\left(x_{1}, \ldots, x_{k}\right) \in R^{k}$ and the parameters $\left(b_{1}, \ldots, b_{k}\right) \in C^{k}$ such that $\operatorname{Re}\left(b_{i}\right)>0, \forall i=1,2, \ldots, k$ and $\mathrm{f}=0$ elsewhere.

The function $\mathrm{B}(\mathrm{b})$ is given in equation (1.11) and

$K\left(x_{1}, \ldots, x_{k} ; z_{1}, \ldots, z_{k}\right)=\left(z_{1} x_{1}^{-1}\right)^{b_{1}} \ldots\left(z_{k} x_{k}^{-1}\right)^{b_{k}}$

The distribution of $\left(z_{1}, \ldots, z_{k}\right)$ has the density

$\bar{f}\left(z_{1}, \ldots, z_{k}\right)=\frac{1}{D_{r_{1}, \ldots, r_{k}}^{m_{1}, \ldots, m_{k}}\left(x_{1}, \ldots, x_{k}\right)} z_{1}^{m_{1}-r_{1}-c_{1}} \ldots z_{k}^{m_{k}-r_{k}-c_{k}}\left(x_{1}-z_{1}\right)^{c_{1}+r_{1}-1} \ldots\left(x_{k}-z_{k}\right)^{c_{k}+r_{k}-1}$

in the region $0 \leq z_{1} \leq x_{1}, \ldots, 0 \leq z_{k} \leq x_{k},\left(m_{i}, r_{i} \in\{0,1,2, \ldots\}\right),\left(m_{i}-r_{i}\right)>0,0<c_{i}<1, \forall i=1, \ldots, k$ and $\overline{\boldsymbol{f}}=0$ elsewhere. The sequence of function $D_{r_{1}, \ldots, r_{k}}^{m_{1}, \ldots, m_{k}}\left(x_{1}, \ldots, x_{k}\right)$ is

$D_{r_{1}, \ldots, r_{k}}^{m_{1}, \ldots, m_{k}}\left(x_{1}, \ldots, x_{k}\right)=\frac{\Gamma\left(c_{1}+r_{1}\right) \Gamma\left(1+m_{1}-r_{1}-c_{1}\right) \ldots \Gamma\left(c_{k}+r_{k}\right) \Gamma\left(1+m_{k}-r_{k}-c_{k}\right)}{\Gamma\left(m_{1}+1\right) \ldots \Gamma\left(m_{k}+1\right)} x_{1}^{m_{1}} \ldots x_{k}^{m_{k}}$

Then, for $\omega=\sqrt{(-1)}$ there exists an expectation formula of an arbitrary function $z_{1}^{-1} \ldots z_{k}^{-1} \Psi\left(z_{1}, \ldots, z_{k}\right)$ with product $x_{1}^{-b_{1}} \ldots x_{k}^{-b_{k}} z_{1}^{b_{1}} \ldots z_{k}^{b_{k}} \hat{\phi}(-\omega, \ldots,-\omega)$ in the form

$\left\langle x_{1}^{-b_{1}} \ldots x_{k}^{-b_{k}} z_{1}^{b_{1}-1} \ldots z_{k}^{b_{k}-1} \hat{\phi}(-\omega, \ldots,-\omega) \Psi\left(z_{1}, \ldots, z_{k}\right)\right\rangle$

$=\frac{\Gamma\left(m_{1}+1\right) \ldots \Gamma\left(m_{k}+1\right)\left(x_{1}^{-1}\right)^{m_{1}+1} \ldots\left(x_{k}^{-1}\right)^{m_{k}+1}}{\Gamma\left(1+m_{1}-c_{1}\right) \ldots \Gamma\left(1+m_{k}-c_{k}\right) Q\left(c_{1}, \ldots, c_{k} ; c_{1}-m_{1}, \ldots, c_{k}-m_{k} ; \alpha_{1}, \ldots, \alpha_{l}\right)}$

$\times H^{\alpha, b, c-m}\left\{e^{z}, \Psi\left(z_{1}, \ldots, z_{k}\right)\right\}\left(x_{1}, \ldots, x_{k}\right)$

Here $H^{\alpha, b, c-m}\left\{e^{z}, \Psi\left(z_{1}, \ldots, z_{k}\right)\right\}$ is defined due to the equation (1.19), $\alpha=\left(\alpha_{1}, \ldots, \alpha_{l}\right) \in C^{l}, l \geq k,\left(\alpha_{i}\right) \neq 0, \forall i=1, \ldots, l$, $c-m=\left(c_{1}-m_{1}, \ldots, c_{k}-m_{k}\right)$, and $0<c_{i}<1$,

$\forall i=1,2, \ldots, k$ and $b=\left(b_{1}, \ldots, b_{k}\right) \in C^{k}$ such that $\operatorname{Re}\left(b_{i}\right)>0, \forall i=1,2, \ldots, k$.

$\hat{\phi}\left(t_{1}, \ldots, t_{k}\right)$ is the characteristic function for the density $f\left(u_{1}, \ldots, u_{k}\right)$ and $\Psi\left(z_{1}, \ldots, z_{k}\right)$ is an arbitrary function and the multiple series 
$Q\left(c_{1}, \ldots, c_{k} ; c_{1}-m_{1}, \ldots, c_{k}-m_{k} ; \alpha_{1}, \ldots, \alpha_{l}\right)=\sum_{r_{1}, \ldots, r_{l}=0}^{\infty} \frac{\left(c_{1}\right)_{r_{1}} \ldots\left(c_{k}\right)_{r_{k}}}{\left(c_{1}-m_{1}\right)_{r_{1}} \ldots\left(c_{k}-m_{k}\right)_{r_{k}}} A_{r_{1}, \ldots, r_{k}}\left(\alpha_{1}\right)^{r_{1}} \ldots\left(\alpha_{k}\right)^{r_{k}}$

Proof: For $\omega=\sqrt{(-1)}$, the characteristic function to the variable $\left(u_{1}, \ldots, u_{k}\right)$ be

(Exton, 1976, p.232;1978, p.130)

$\hat{\phi}\left(t_{1}, \ldots, t_{k}\right)=\int_{-\infty}^{\infty} .(k) \cdot \int_{-\infty}^{\infty} \exp \left[\omega\left(t_{1} u_{1}+\ldots+t_{k} u_{k}\right)\right] f\left(u_{1}, \ldots, u_{k}\right) d u_{1} \ldots d u_{k}$

where $f\left(u_{1}, \ldots, u_{k}\right)$ is the density function.

Now, make an appeal to the equations (5.1) and (5.2) in equation (5.7), we find

$$
\begin{aligned}
x_{1}^{-b_{1}} \ldots x_{k}^{-b_{k}} z_{1}^{b_{1}} & \ldots z_{k}^{b_{k}} \hat{\phi}(-\omega, \ldots,-\omega)=\frac{z_{k}^{b_{k}} x_{k}^{-b_{k}}}{B(b)} \int \ldots \int \exp \left[u_{1}+\ldots+u_{k}\right] u_{1}^{b_{1}-1} \ldots u_{k-1}^{b_{k-1}-1} \\
& \times\left(1-\frac{u_{1} x_{1}}{z_{1}}-\ldots-\frac{u_{k-1} x_{k-1}}{z_{k-1}}\right)^{b_{k}-1} d u_{1} \ldots d u_{k-1}
\end{aligned}
$$

Thus, an expectation formula of an arbitrary function $z_{1}^{-1} \ldots z_{k}^{-1} \Psi\left(z_{1}, \ldots, z_{k}\right)$ with product of $x_{1}^{-b_{1}} \ldots x_{k}^{-b_{k}} z_{1}^{b_{1}} \ldots z_{k}^{b_{k}} \hat{\phi}(-\omega, \ldots,-\omega)$ may be found by (Exton, 1976, p.220)

$$
\begin{aligned}
& \left\langle x_{1}^{-b_{1}} \ldots x_{k}^{-b_{k}} z_{1}^{b_{1}-1} \ldots z_{k}^{b_{k}-1} \hat{\phi}(-\omega, \ldots,-\omega) \Psi\left(z_{1}, \ldots, z_{k}\right)\right\rangle=\frac{x_{k}^{-b_{k}}}{B(b) D_{r_{1}, \ldots, r_{k}}^{m_{1}, \ldots, m_{k}}\left(x_{1}, \ldots, x_{k}\right)} \int_{0}^{x_{1}} \ldots \int_{0}^{x_{k}}\left(x_{1}-z_{1}\right)^{c_{1}+r_{1}-1} . . \\
& \times \ldots\left(x_{k}-z_{k}\right)^{c_{k}+r_{k}-1} z_{1}^{m_{1}-r_{1}-c_{1}-1} \ldots z_{k-1}^{m_{k-1}-r_{k-1}-c_{k-1}-1} z_{k}^{b_{k}+m_{k}-r_{k}-c_{k}-1} \int \ldots \int \exp \left[u_{1}+\ldots+u_{k}\right] u_{1}^{b_{1}-1} \ldots u_{k-1}^{b_{k-1}-1} \\
& \times\left(1-\frac{u_{1} x_{1}}{z_{1}}-\ldots-\frac{u_{k-1} x_{k-1}}{z_{k-1}}\right)^{b_{k}-1} d u_{1} \ldots d u_{k-1} \Psi\left(z_{1}, \ldots, z_{k}\right) d z_{1} \ldots d z_{k}
\end{aligned}
$$

Thus, in both sides of equation multiply

$D_{r_{1}, \ldots, r_{k}}^{m_{1}, \ldots, m_{k}}\left(x_{1}, \ldots, x_{k}\right) \frac{x_{1} \ldots x_{k}}{\Gamma\left(c_{1}\right) \ldots \Gamma\left(c_{k}\right)} A_{r_{1}, \ldots, r_{k}}\left(\alpha_{1}\right)^{r_{1}} \ldots\left(\alpha_{l}\right)^{r_{l}}$ and then on defining by equation (5.4) sum $\mathrm{r}_{1}, \ldots, \mathrm{r}_{1}$ respectively from

0 to $\infty$ and again make an appeal to the formula given in equation (1.19) with the definitions given therein and the multiple series given in equation (5.6), finally, we evaluate equation (5.5).

\section{Conclusions}

The operators

$H^{\alpha, b, c}\left\{g(z), \Psi\left(z_{1}, \ldots, z_{k}\right)\right\}\left(x_{1}, \ldots, x_{k}\right)$ and $\mathrm{P}^{\alpha, b, c}\left\{g(z), \Psi\left(z_{1}, \ldots, z_{k}\right)\right\}\left(x_{1}, \ldots, x_{k}\right)$ defined by (1.19) and (1.20) are the generalized fractional integral operators involving Dirichlet averages and any general special function. They, particularly, give Saigo and Maeda operators (see,equations (1.21)-(1.24)). They are identical to series of successive Erde'lyi-Kober operators of any arbitrary function (see, equations (2.1), (2.3) and (2.5)). An application of these operators give the analytic images of the general class of multivariable polynomials due to Srivastava and Garg with product of exponential functions (see, section-3).The formula of any arbitrary function $z_{1}^{-1} \ldots z_{k}^{-1} \Psi\left(z_{1}, \ldots, z_{k}\right)$ with product of $\left(z_{1} x_{1}^{-1}\right)^{b_{1}} \ldots\left(z_{k} x_{k}^{-1}\right)^{b_{k}}$ and the characteristic function of Dirichlet density at the point $(-\omega, \ldots,-\omega)$ equals the operator $H^{\alpha, b, c}\left\{g(z), \Psi\left(z_{1}, \ldots, z_{k}\right)\right\}\left(x_{1}, \ldots, x_{k}\right)$ when $g(z)=e^{z}$ (see, section-5). With the help of identities given in this paper, we may evaluate other images of the general class of multivariable polynomials with products of the exponential functions in terms of the multiple series consisting general coefficients and other multivariable special functions and polynomials. 


\section{Aknowledgement}

The authors are grateful to the referees for their valuable suggestions for the improvement of this paper.

\section{References}

Appell P. and Kampe' de Fe'riet J., 1926. Fonctions Hyperge'ome'triques et Hyperspheriques Polynōmes d'Hermite, GauthierVillars, Paris.

Carlson B. C., 1977. Special Functions of Applied Mathematics, Academic Press, New York.

Erde'lyi et al. A., 1953. Higher Transcendental Functions, Vol. I McGraw - Hill Book Co., New York.

Gupta S. G. and Agrawal B. M., 1990. Dirichlet averages and fractional derivatives, J. Indian Acad. Math. Vol. 12, No. 1, pp.103115.

Exton H.,1976. Multiple Hypergeometric Function and Application, John Wiley and Sons, New York.

Exton H., 1978. Hand Book of Hypergeometric Integrals : Theory, Applications, Tables, Computer Programs, Ellis Harwood Limited, New York.

Kilbas A.A., Srivastava H.M. and Trujillo J.J., 2006 . Theory and Applications of Fractional Differential Equations, Elsevier Inc., New York.

Kiryakova V., 2006. On two Saigo's fractional integral operators in the class of univalent functions, FCAA, Vol. 9, No. 2, p.p. 159176.

Lauricella G., 1893. Sulle funzioni ipergeometriche a pì̀ variabili”, Rend. Circ. Mat. Palermo, Vol. $7,111-158$.

Mahtai A. M. and Saxena R. K., 1973. Generalized hypergeometric functions with applications in statistics and physical sciences, Lecture Notes in Mathematics, Vol. 348, Springer Verlag, Heidelberg.

Rainville E. D., 1971. Special Functions, McMillan Co. Inc. New York, 1960; Reprinted by Chelsea Publ. Co. Bronx, New York.

Saigo M., 1978. A remark on integral operators involving the Gauss hypergeometric functions, Math. Rep. Kyushu. Univ. 11, pp. 135-143.

Saigo M. and Maeda N., 1996. More generalizations of fractional calculus, Transform Methods and Special Functions, Varna, Bulgaria, pp. 386-400.

Samko S. G. , Kilbas A. A. and Marichev O.I., 1993. Fractional Integrals and Derivatives Theory and Applications, Gordon and Breach.

Srivastava H. M., 1972. A contour integral involving Fox's H-function, Indian J. Math., Vol. 14 pp. 1-6.

Srivastava H. M. and Garg M., 1987. Some integrals involving a general class of polynomials and the multivariable H-function, Rev. Roumaine Phy. Vol. 32, pp.685-692.

Srivastava H. M., Gupta K. C. and Goyal S. P., 1982. The H-Function of One and Two Variables with Applications, South Asian Publishers, New Delhi and Madras.

Srivastava H. M., and Manocha H. L., 1984. A Treatise on Generating Functions, Ellis Horwood Limited, New York.

Srivastava H. M. and Panda R. 1976a. Some bilateral generating functions for a class of Generalized hypergeometric polynomials, J. Reine Angew. Math. Vol. 283/284, pp. 265-274.

Srivastava H. M. and Panda R., 1976b. An integral representation for the product of two Jacobi polynomials, J. London Math. Soc. Vol. 2, No. 12, pp. 419-425.

\section{Biographical notes}

Prof. M. A. Pathan has served as Chairman of the Department of Mathematics, Aligarh Muslim University, Aligarh, India. Presently, he is working in the Department of Mathematics, University of Botswana, Gaborone, Botswana. He has supervised 13 Ph.D's in Integral operators, Fractional calculus, Lie theory and Special functions. He wrote 4 books and published more than 140 research papers. He is former President of the Indian Mathematical Society, Sectional President of Indian Science Congress Association and is General Secretary of the Society for Special Functions and their Applications. He is on the Editorial board of more than 10 Journals,/Bulletins and Proceedings of international conferences.

Dr. Hemant Kumar has received Ph. D. Degree in 1993 from Burkatullah University, Bhopal, (M. P.), India. He is an Associate Professor in the Department of Mathematics, D. A-V. P. G. College, Kanpur, (U. P.), India. He has more than 20 years of research experience. His research interests include Mathematical Modeling and Special functions, Application of Special functions in Boundary value problems, Fractional Calculus and Generating functions via Lie Groups. He has published more than 30 papers in national and international journals. He has also presented more than 35 research articles in national and international conferences.

Shilesh Kumari is a research scholar doing her Ph. D. from Chhatrapati Sahu Ji Maharaj University, Kanpur, (U. P.), India under the supervision of Dr. Hemant Kumar, Associate Professor, Department of Mathematics, D. A-V. P. G. College, Kanpur, (U. P.), India. She has four years research experience.

Received March 2010

Accepted April 2010

Final acceptance in revised form June 2010 